\title{
BactoSim - An Individual-Based Simulation Environment for Bacterial Conjugation
}

\author{
Antonio Prestes García and Alfonso Rodríguez-Patón \\ Departamento de Inteligencia Artificial, \\ Universidad Politécnica de Madrid, Campus de Montegancedo s/n, \\ Boadilla del Monte, 28660 Madrid, Spain \\ antonio.garcia2@gmail.com
}

\begin{abstract}
BactoSim is an agent-based platform for simulating the conjugation in spatially structured bacterial populations, which are the conditions typically found on naturally occurring colonies such as biofilms or in agar-based laboratory cultures. The model provides a set of key indicators which can be visualized in real time as the simulation evolves and saved as for further analysis.
\end{abstract}

\section{Introduction}

The bacterial conjugation is a natural process where bacterial cells interchange circular DNA fragments known as plasmids. Conjugative plasmids are the vehicles of antibiotic resistance spreading in hospitals and also may carry genes which are responsible for bacterial virulence and pathogenicity [2]. Therefore it is worth to understand how they are propagated and that is the key point where individual-based models come to help to shed light over the inner intricacies of the process. The most common strategy for modeling conjugation was some variation of differential equations with mass action kinetics using whole population data for model calibration. But one of the main drawbacks of this approach is that it fails to take into account local variations and assume wellmixed environments which clearly are not realistic assumptions. In order to produce a more structurally realistic representation for conjugation, a spatially explicit individual-based model is required where agents have their own individual internal state and interact only with their closest neighbors being the changes in spatial position of agents exclusively consequence of the shoving relaxation due to the colony growth process. The BactoSim code and binaries are available for download ${ }^{1}$. The simulator is part of project European project PLASWIRES which also includes the simulator BactoSim II [1].

\section{Main Purpose}

In this paper we provide a brief overview of the BactoSim simulation platform. The main objective of the software is to provide a computational workbench for

1 http://goo.gl/TDGxNr 
using bacterial plasmid as a "wiring protocol" for harnessing the power of bacterial cell-cell communication as a tool for multicellular synthetic biology. This is achieved using an integrative approach where molecular level and individualbased observations are incorporated within the simulation model. Hence all relevant data about the system under study is used as model parameters and to implement the model rule base. This is a bottom-up approach where we specify how agents must evolve thereby producing an emergent global behavior. That kind of models is also useful for producing new insights about the process being studied, suggesting sometimes counter intuitive ideas about what local processes are responsible for some global system comportment. The simulation software was developed using the Repast Symphony multi-agent simulation platform [3].

\section{Demonstration}

The simulation model is comprised by a discrete set of agents each of them representing a single bacterial cell $\beta_{i}, i=1 \ldots N$ where $N$ is the current population size at the simulated time $t$. The agents interact with other agents and with the environment which holds some amount of nutrient. The environment is implemented using a value layer of repast framework [3]. The $\beta_{i}$ agents lives and evolve in a computational domain represented as a $1000 \times 1000$ discrete $^{2}$ grid, corresponding to a real surface of $1 \mathrm{~mm}^{2}$. In approximately 20 minutes of wall clock, Bactosim is able to simulate 600 minutes of colony growth with a final population of $10^{5}$ bacterial cells.

The bacterial cells evolve during the simulated time having their state variables updated by some processes representing the intra and intercellular behavior of every agent $\beta_{i}$. Hence, at a functional high level, the model have a set of processes $\mathcal{P}=\left(p_{1} \ldots p_{5}\right)$ each of them standing respectively for the Uptake, Diffusion, Division, T4SS expression (a protein needed for plasmid conjugation), Shoving relaxation and finally the Conjugation. The execution of these processes is shuffled in order to avoid any bias and the state of agents and the value layer holding the nutrient particles are updated asynchronously. The bacterial cells have three different states, namely $R, D$ and $T$ standing respectively for plasmid free (or recipient cells), plasmid donors (cells originally infected by plasmid) and transconjugant cells which are those plasmid free cells that have been infected by a donor or transconjugant cell.

The model requires the input of two groups of parameters, the first group is related to the definition of virtual plasmid-host features which will be simulated and the second group allows the specification of the initial population sizes. The first group includes the parameters $G$ which is the doubling time for plasmid free cells $^{3}$, the point of cell cycle where conjugation is deemed most prone to happen [4], the T4SS expression and the conjugation cost. The value of $\gamma_{0}$ must be also introduced, that parameter tells the model how many conjugations, on average,

${ }^{2}$ Actually it is implemented as a repast multiOccupancy2D allowing cells to overlap in some extent, in the same way it really happens in a real bacterial colony.

${ }^{3}$ The values of doubling time for $D$ and $T$ cells are both emergent properties of model. 


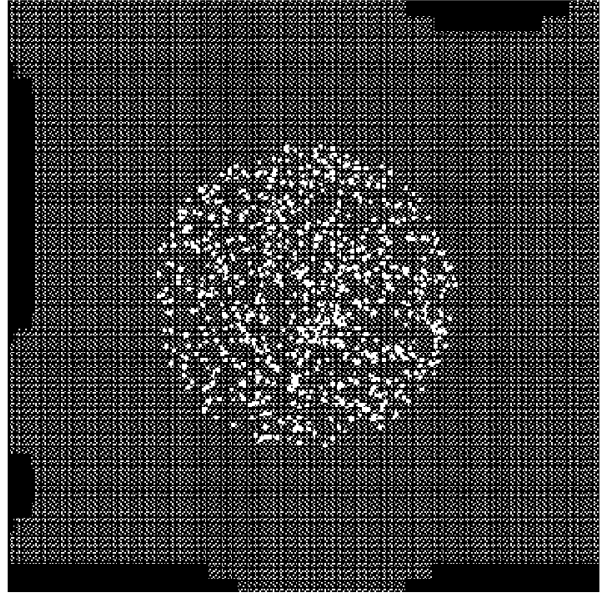

(a) Initialization

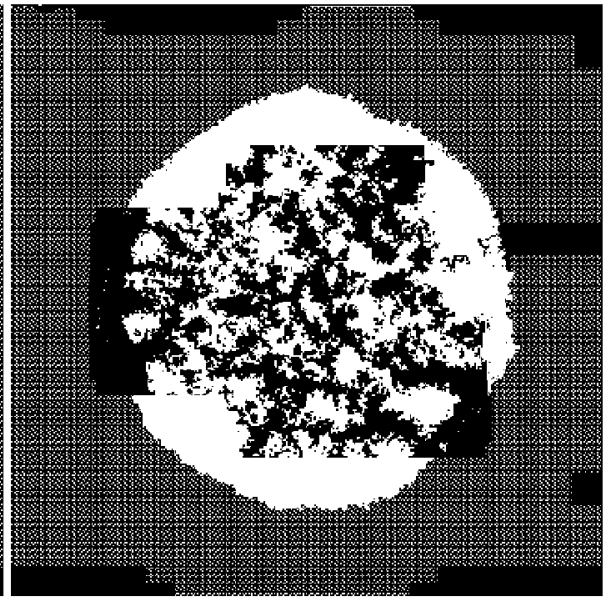

(b) $t=600$ minutes

Fig. 1. The BactoSim Virtual Agar-Plate view for simulated version of $R 1$ plasmid. The color scheme used is green for $R$ cells, red for $D$ cells and blue for $T$ cells. The light-green, light-read and light-blue colors are used to represent depleted nutrient zones where cells are no longer dividing.

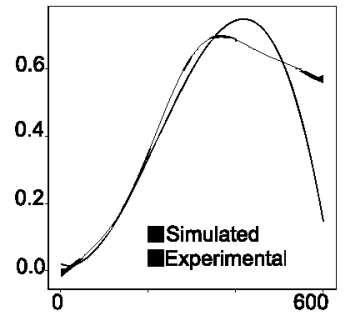

(a) $T /(T+R)$

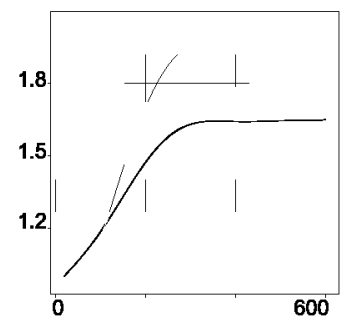

(d) $\gamma_{0}$

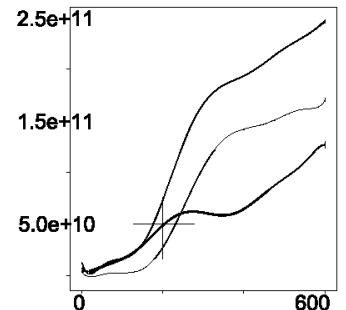

(b) Population

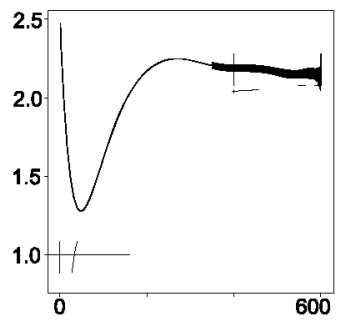

(e) $R_{0}$

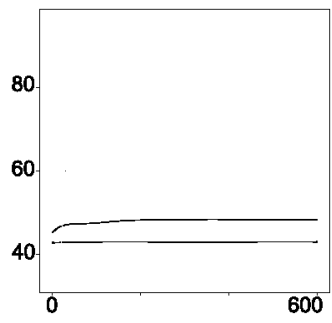

(c) Doubling time

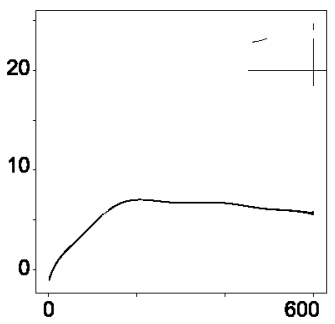

(f) $\%$ Horizontal

Fig. 2. Sample of the output provided by the BactoSim model 
are performed by bacterial cells. Model requires also two flags which allow the definition of repressed and conjugative plasmids.

The second group of parameters includes the value of $N_{0}$ or the initial population size which must be introduced in the model as a concentration in cells $/ \mathrm{ml}$ and the initial proportion of donor cells as a percentage of $N_{0}$. Besides of these two groups the model has an optional parameter for a polynomial equation fitted to the experimental data which serve as a simple visual assessment of the quality of simulated data, an example of this can be seen in Figure $2 \mathrm{a}$.

The model generates a lot of output with both quantitative and qualitative data about the evolution of the agents and the colony global outcomes. With respect to qualitative data a sample output is show in Figure 1 which represents, in some extent, a virtual agar-plate where bacterial agents interact and evolve during the simulated time.

The virtual-plate provides a simple visual tool to assess whether the colony is growing in a realistic pattern. Besides of the "plate view", BactoSim generates six performance indicators shown in Figure 2: the $T /(T+R)$ rate in Figure $2 \mathrm{a}$, the population size and the doubling time in Figure $2 \mathrm{~b}$ and $2 \mathrm{c}$, the $\gamma_{0}$, $R_{0}$ (Basic Reproduction Number) and the ratio of new infections caused by horizontal transfers in Figures 2d, 2e and $2 \mathrm{f}$ respectively

\section{Conclusions}

In this paper we have presented the main features of BactoSim simulation environment and the master lines which have been used to implement the conjugation model and it is worth to mention that it is still an ongoing process. As has been outlined the objective of our model is twofold, on the one hand it is intended to be a predictive tool and on the other hand the model is a helpful tool to gather insights on the intra-cellular process which are building the global dynamics of the conjugation cell-cell communication at a whole colony level.

Acknowledgments. This work was supported by the European FP7 - ICT - FET EU research project 612146 and by MINECO research grant TIN2012-36992.

\section{References}

1. Beneš, D., Sosík, P., Rodríguez-Patón, A.: An Autonomous In Vivo Dual Selection Protocol for Boolean Genetic Circuits. Artificial life, 1-14, January 2015. http:// view.ncbi.nlm.nih.gov/pubmed/25622012

2. Norman, A., Hansen, L.H., Sørensen, S.J.: Conjugative plasmids: vessels of the communal gene pool. Philosophical transactions of the Royal Society of London. Series B, Biological Sciences 364(1527), 2275-2289 (2009). http://dx.doi.org/10.1098/rstb. 2009.0037 
3. North, M., Collier, N., Ozik, J., Tatara, E., Macal, C., Bragen, M., Sydelko, P.: Complex adaptive systems modeling with Repast Simphony. Complex Adaptive Systems Modeling 1(1), 1-26 (2013). http://dx.doi.org/10.1186/2194-3206-1-3

4. Seoane, J., Yankelevich, T., Dechesne, A., Merkey, B., Sternberg, C., Smets, B.F.: An individual-based approach to explain plasmid invasion in bacterial populations. FEMS Microbiology Ecology 75(1), 17-27 (2011). http://dx.doi.org/10.1111/ j.1574-6941.2010.00994.x 